\title{
Possible chemical mimicry of the European lady's slipper orchid (Cypripedium calceolus)
}

Tomasz Przybyłowicz' ${ }^{1}$, Peter Roessingh ${ }^{1}$, Astrid T. Groot ${ }^{1}$, J.C. (Koos) Biesmeijer ${ }^{2}$, J.G.B. (Gerard) Oostermeijer ${ }^{1}$, Lars Chittka ${ }^{3}$, Barbara Gravendeel ${ }^{1,2,4}$

${ }^{1}$ Institute for Biodiversity and Ecosystem Dynamics (IBED), Universiteit van Amsterdam, PO Box 94248, 1090 GE Amsterdam, The Netherlands

${ }^{2}$ Netherlands Centre for Biodiversity (NCB) Naturalis, PO Box 9500, 2300 RA Leiden, The Netherlands

${ }^{3}$ School of Biological and Chemical Sciences, Queen Mary University of London, Mile End Road, London E1 $4 N S, U K$

${ }^{4}$ E-mail: barbara.gravendeel@ncbnaturalis.nl

Key words: bees, behaviour, chemical mimicry, deception, EAG, pollination

\begin{abstract}
Pollination based on insect deception has been debated in the scientific community since it was first reported over two hundred years ago. A vast majority of deceptive syndromes occur within the orchid family. While many cheating flowers have been described and are well known, there are still many curious cases that need further investigation. One prime example of such a case is Cypripedium calceolus, known as European lady's slipper orchid. While the flower has been of interest to many prominent scientists for over a century, its pollination is still not fully understood. Both visual and olfactory cues seem to play an important role in pollinator attraction. In this study we focussed on the olfactory cues in order to explore their relationship (in future experiments) with floral visual cues, including the unique asymmetry of these flowers. Some of the plants' floral fragrances were used in Electroantennography experiments. Eleven chemical compounds were applied to the antennae of Bombus terrestris and Apis mellifera. Even though these species are not regular visitors of $C$. calceolus, we were interested to see whether there were common principles in their responses to the flowers' scent that might justify extrapolating to other pollinator species such as sand bees that get trapped in these orchids and fly out of the flowers afterwards with pollen smeared on their body. The results show that while both species react similarly to most of the odours, some of the tested acetates induced a significantly greater reaction in B. terrestris antennae. These acetates play an important role in bumblebee pheromones, but their relevance for the natural pollinators of C. calceolus remains to be confirmed to see whether chemical mimicry by these flowers is deliberately employed to attract pollinators.
\end{abstract}

\section{Contents}

Introduction 103

Material and methods 105

Bumblebee and honeybee workers'

antenna preparation

Preparation of the stimuli for the experiment

Electorantennography

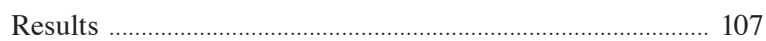

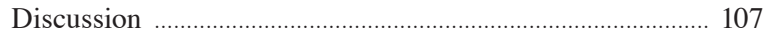

Conclusions and further study ............................................... 108

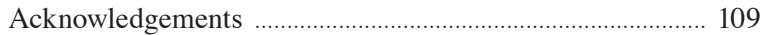

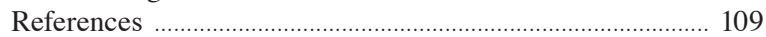

\section{Introduction}

Deception in pollination by orchids was first reported by Sprengel (1793), who noticed that some of the species within the genus Orchis do not produce any nectar. Interestingly, he observed that those flowers were nonetheless visited by insects. At the beginning, this novel idea was met with disbelief (Dafni, 1984), even by Darwin (1877), who wrote: “...[I]t appears to me incredible that the same insect should go on visiting flower after flower of these orchids, although it never obtains any nectar. Insects, or at least bees, are by no means destitute of intelligence." Today, the occurrence of deception in the pollination of many plant species is widely accepted. While it is not limited to orchids (Jersáková et al., 2006; Schiestl, 2005; 2010), it occurs in the orchid family at rates unparalleled in any other plant family (Scopece et al., 2010). It is estimated that about one-third of all orchids deceive their pollinators (Ackerman, 1986). However, in many individual species details of the mechanisms by which pollinators are deceived are still lacking. Many studies on the subject call for further research (Ferdy et al., 1998; Cozzolino and Widmer, 2005; Bell et al., 2009) and a multi-disciplinary approach (Dafni, 1984).

The European lady's slipper orchid (Cypripedium calceolus $\mathrm{L}$.) is an example which illustrates this deficiency. Despite the fact that $C$. calceolus has been 
studied for well over a century (Darwin, 1877), and is native to Europe, its pollination is still poorly understood (Case and Bradford, 2009). Flowers of C. calceolus are characterized by a large yellow pouch which is a modified labellum. The pouch has an opening on the top and its rim is covered by a slippery substance. Insects that land on the top of the labellum are very likely to slip inside the pouch where they are trapped. To free itself, an insect has to use one of the two orifices in the back of the pouch. During the laborious process of crawling out, the pollen mass is smeared on the dorsal side of the bodies of visitors with the correct size. The same insect has to fall into another C. calceolus flower to ensure pollination. A visitor that is too big has a chance of climbing out of the pouch without touching any reproductive parts of the flower (Nilsson, 1979; Erneberg and Holm, 1999; Claessens and Kleynen, 2011).

Insects, especially bees, can learn to avoid unrewarding flowers (Dafni, 1984; Dyer and Chittka, 2004; Lotto and Chittka, 2005; Benard et al., 2006; Raine and Chittka, 2008). Therefore, the discussion on reproduction of $C$. calceolus is dominated by research aimed to discern all the cues that attract bees to the flowers. The discussion on possible attractants is still ongoing and is far from resolved. Müller (1873) was one of the first to suggest that both visual and olfactory cues play an important role in insect attraction to slipper orchids. The first experimental evidence for the presence of visual cues was provided by Daumann (1968) who removed the yellow pouch and discovered that bees were no longer attracted. Welch $(1998,2002)$ analyzed asymmetry of slipper orchid flowers and discovered that various Cypripedium species among which $C$. calceolus have tepals all coiling in the same direction. This makes the flowers asymmetrical. The coiling tepals might have a special function in insect deception by distorting floral symmetry. Bees have more difficulty memorizing asymmetrical flowers than symmetrical ones (Giurfa et al., 1999; Rodríguez et al., 2004). The coiled tepals of rewardless slipper orchid flowers might make it more difficult for bees to recognize and avoid these flowers during foraging. Nilsson (1979) brought attention to olfactory cues and suggested that undulating flight patterns of the bees approaching a slipper orchid flower resulted from chemical attraction. The theory of a strong role of olfactory cues in this species gained wide acceptance (Bergström et al., 1992; Barkman et al., 1997).

With the introduction of the Gas ChromatographyMass Spectrometry technique (GC-MS), the composi- tion of the scent of C. calceolus was determined (Nilsson, 1979; Bergström et al., 1992; Barkman et al., 1997). These studies were the first to unravel floral fragrance composition of European lady's slipper orchid and its close American relatives. It contains large amounts of acetates - chemical compounds that are also found in pheromones used by many bees, including Andrena bees (Tengö and Bergström, 1977) and bumblebees (Appelgren et al., 1991; Bertsch et al., 2004; Coppée et al., 2008). Some of the other compounds present in Cypripedium flowers include alphafarnesene, nerol and linalool, which are common plant compounds also found in bee secretions, where they were found to be associated with receptive females of solitary bees (Cane and Tengo, 1981) and nest odour of solitary bees (Barkman et al., 1997) and bumblebees (Granero et al., 2005). Bombus terrestris (Linnaeus, 1758) pheromones employed to recruit foragers to a food source include many of the same chemical compounds found in lady's slipper orchid's scent. Granero et al. (2005) recognized 11 volatile chemical compounds in this particular pheromone. Seven of those ( $\alpha$-pinene, $\beta$-pinene, myrcene, limonene, ocimine, linalool and nerol) are found in C.calceolus scent as well (Bergström et al., 1992, Barkman et al., 1997). Linalool and nerol are also found in nest odour of bumblebees and Andrena species (Barkman et al., 1997; Granero et al., 2005). These findings are consistent with the idea that orchids evolved in response to instinctive behaviours of their visitors. In short, to successfully and repeatedly 'cheat' an insect equipped with keen learning abilities such as most species of bees (Chittka et al., 1992; Waser et al., 1996), the cue has to induce innate behaviour that the visitor will have difficulty resisting (Jersáková, 2006).

Most of the olfactory studies so far focused on revealing the chemical composition of the floral fragrance of the European lady's slipper orchid (Nilsson, 1979; Bergstrom et al., 1992; Barkman et al., 1997), occasionally comparing it to chemical compounds known to be produced by insects (Claessens and Kleynen, 2011). Such an approach can provide valid information about the possible role of flower stimuli in attracting pollinators. This research aimed to combine previously separately analyzed chemical compounds from either slipper orchid flowers or bees, in a single practical experiment with real sensory systems responsible for insect attraction, using electroantennography (EAG). This bioassay technique is based on the electric depolarisation of the olfactory neurons that creates measurable voltage fluctuations. In other 
words, when a volatile stimulus is presented to an insect and the chemical binds to the sensory cells in the antenna, it creates changes in the electrical potential, which can be recorded (Schneider, 1957). Once more is known about olfactory cues employed by C. calceolus in pollinator attraction, their interaction with visual cues (including the unusual asymmetry of the display apparatus in this species) can be investigated.

\section{Material and methods}

\section{Bumblebee and honeybee workers' antenna prepara- tion}

European honeybees (Apis mellifera (Linnaeus, 1758)) and bumblebees (Bombus terrestris) were used for the experiment. These bees are not common visitors of $C$. calceolus, but they are more readily available for laboratory studies than many of the solitary species that are often trapped by this slipper orchid. Since some sensory systems (for example colour vision; see Chittka, 1996) are highly conserved across bee species, we sought to explore whether honeybees and bumblebees might display similar chemosensory responses to orchids' scent components; this would perhaps allow us to extrapolate to the responses of other bee species until they become available for experimentation (Baer, 2003). Eleven honeybee workers and ten bumblebee workers were tested. Honeybees were collected at the entrance of a private beehive in Diemerpark, Amsterdam-Oost, on the day of the experiment. Bumblebees were obtained from a commercial bumblebee colony (Koppert Biological Systems) that was kept in the lab. They had an access to the flight cage where they were provided with $50 \%$ sucrose solution presented on yellow plastic discs $2.5 \mathrm{~cm}$ in diameter. A 15W UV light was used to mimic daylight and aid in the foraging of the bees.

Right before EAG reading, individual bees were put in a Petri dish and anaesthetized with $\mathrm{CO}_{2}$ for 10 seconds. Next, the head was cut off with a surgical scalpel and a sharp glass capillary filled with insect ringer solution was inserted into the brain via the foramen magnum. A thin silver wire was put inside the capillary and connected to an amplifier. Next, the tip of the right antenna (one segment) was cut off. It was placed inside the second capillary prepared in a similar manner as the first one. The second silver wire (ground) was placed inside. Great care was taken to ensure that only the very end of the antenna came into contact with the ringer solution inside the capillary. This maximized the amount of the sensory cells exposed to the air. The setup was positioned in such a way that the antenna was less that $1 \mathrm{~cm}$ away from the end of the air flow tube.

\section{Preparation of the stimuli for the experiment}

The compounds used in the experiment were chosen based on two factors. The first was their abundance in slipper orchid floral fragrances - the Eurasian Cypripedium calceolus and its two North American varieties Cypripedium calceolus L. pubescens (Willd.) Correll and Cypripedium calceolus L. parviflorum (Salisb.) Fernald, according to Bergström et al. (1992). From this study, $\alpha$-pinene (Fluka, $\geq 99 \%$ purity), caryophyllene (Sigma, $\geq 98.5 \%$ purity), cis- $\beta$ ocimene (Fluka, $70 \%$ purity with $25 \%$ limonene), decyl acetate (Alfa Aesar, 98\% purity ), dodecyl acetate (Aldrich, 97\% purity), linalool (Fluka, 97\% purity), octyl acetate (Aldrich, $99 \%$ purity), trans- $\beta$ farnesene (Max Planck Institute for Chemical Ecology, 98\% purity) and trans- $\beta$-ocimene (RC Treatt, $\sim 97 \%$ purity) were selected. The second criterion was based on additional studies of Barkman et al. (1997). Based on this study, nerol and nonanal were selected. In total, eleven chemical compounds were chosen based on availability in our lab. Stimuli were prepared by diluting standard stock chemical compounds in dichloromethane $\left(\mathrm{CH}_{2} \mathrm{Cl}_{2}\right)$ to concentrations of $1.0 \times 10^{-1}$, $1.0 \times 10^{-2}$ and $1.0 \times 10^{-3}$. Green plant volatile (Z-3-Hexen-1-ol) in $1.0 \times 10^{-2}$ concentration was prepared in the same way as the rest of the stimuli and was used as an internal standard during the EAG measurements, as the response of the antenna diminishes with time. The control consisted of pure dichloromethane and it was prepared and applied together with other compounds. A volume of $20 \mu \mathrm{L}$ of each sample was then put on 5 $\mathrm{mm} \times 50 \mathrm{~mm}$ filter paper and placed inside a Pasteur pipette. This resulted in a total of 35 stimuli. All dilutions were prepared before the experiments and kept in glass bottles with Teflon ${ }^{\circledR}$ lined caps. Pasteur pipettes were prepared daily.

\section{Electorantennography}

The chemical stimuli were injected into the main air flow $(3300 \mathrm{ml} / \mathrm{min})$ by switching a stimulus flow for one second from an empty Pasteur pipette through a pipette containing the stimulus, resulting in a $50 \mathrm{ml}$ odour pulse. Stimuli were applied with 30 second intervals. 
All 11 compounds in three concentrations created a total of 33 samples that were randomized using Research Randomizer (v. 3.0) by Urbaniak and Plous (2011). Each run was started by the internal standard (Z-3-Hexen1-ol) followed by the control. Next, 16 samples were tested. A series of standard, control and standard was used in the middle of the experimental run. It was followed by another 17 samples. The experiment was finished by the control and the standard sample.

The amplitude of the EAG was measured with an IDAC-4 amplifier and recorded with EAG2000 software (both available from Syntech, Kirchzarten,
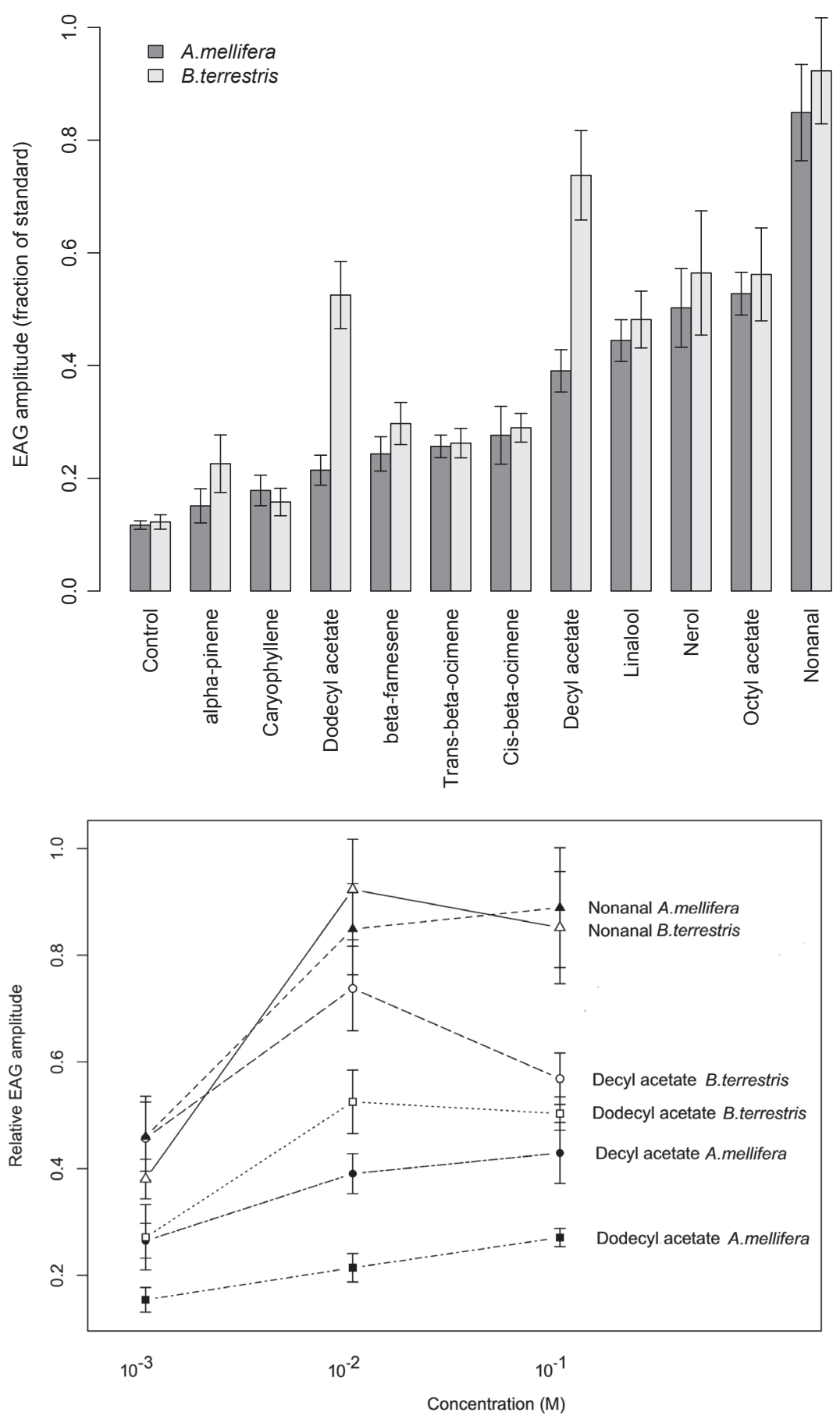

Fig. 1. Results of the EAG experiment. Antennal reactions of $A$. mellifera and B. terrestris toward $20 \mu 1$ of a $0.01 \mathrm{M}$ solution of the 11 chemical compounds tested. The response is expressed as a fraction of the response to the standard cis-3-hexen-1-ol.
Fig. 2. Dose response curves of EAG responses for nonanal, decyl acetate and dodecyl acetate in workers of A. mellifera and B. terrestris. The two acetates induced significantly higher responses in B. terrestris. Responses are expressed as fractions of the response to the standard cis-3-hexen-1-ol. 
Table 1.

Analysis of variance of relative EAG responses as a function of species, stimulus, and stimulus concentration. In addition to a significant effect of concentration, a significant interaction between species and stimulus was found. Significance code: $<0.01=* *$

\begin{tabular}{|c|c|c|c|c|c|c|}
\hline Effect & Df & Sum Sq & Mean Sq & $F$ value & $\operatorname{Pr}(>F)$ & \\
\hline Species & 1 & 2.740 & 2.740 & 15.5466 & $<0.001$ & $* *$ \\
\hline Stimulus & 11 & 191.800 & 17.436 & 98.9418 & $<0.001$ & $* *$ \\
\hline Concentration & 1 & 42.418 & 42.418 & 240.6969 & $<0.001$ & $* *$ \\
\hline Species : stimulus & 11 & 8.263 & 0.751 & 4.2625 & $<0.001$ & $* *$ \\
\hline Residuals & 693 & 122.127 & 0.176 & & & \\
\hline
\end{tabular}

Table 2. Significance of coefficients for the species-stimulus interaction. Only the two acetates differed between the two species. Significance codes: $<0.001=* * *,<0.05=*$

\begin{tabular}{|c|c|c|c|c|c|}
\hline Interaction & Estimate & Std. Error & $\mathrm{t}$ value & $\operatorname{Pr}(>|t|)$ & \\
\hline B.terrestris: B-farnesene & -0.076 & 0.155 & -0.491 & 0.624 & \\
\hline B. terrestris: Caryophyllene & -0.251 & 0.153 & -1.641 & 0.101 & \\
\hline B. terrestris: Cis-B-ocimene & -0.072 & 0.154 & -0.465 & 0.642 & \\
\hline B. terrestris: Decyl acetate & 0.367 & 0.155 & 2.364 & 0.018 & $*$ \\
\hline B. terrestris: Dodecyl acetate & 0.579 & 0.155 & 3.745 & 0.000 & $* * *$ \\
\hline B. terrestris: Linalool & 0.049 & 0.154 & 0.32 & 0.749 & \\
\hline B. terrestris: Nerol & 0.041 & 0.154 & 0.269 & 0.788 & \\
\hline B. terrestris: Nonanal & -0.117 & 0.154 & -0.76 & 0.448 & \\
\hline B. terrestris: Octyl acetate & 0.002 & 0.153 & 0.013 & 0.989 & \\
\hline B. terrestris: Standard & -0.096 & 0.153 & -0.628 & 0.530 & \\
\hline B. terrestris: Trans-B-ocimene & -0.025 & 0.154 & -0.16 & 0.873 & \\
\hline
\end{tabular}

Germany). The responses to the standards were interpolated to calculate a theoretical standard response at the time of each stimulus and the observed EAGs were expressed relative to this standard. These relative amplitudes were $\log$ transformed to normalise the response distribution and were analysed using a linear model with main effects for stimulus, concentration and species. Since we were primarily interested in species differences, we also included the interaction between species and stimulus. All statistical tests were performed in R, version 2.13.1 (Hornik, 2011).

\section{Results}

All synthetic samples of the volatiles found in $C$. calceolus ( $\alpha$-pinene, caryophyllene, cis- $\beta$-ocimene, decyl acetate, dodecyl acetate, linalool, nerol, nonanal, octyl acetate, trans- $\beta$-farnesene and trans- $\beta$-ocimene) induced EAG responses in the antennae of female A. mellifera $(\mathrm{n}=11)$ and $B$. terrestris $(\mathrm{n}=10)$. The response to the standard (Z-3-Hexen-1-ol) was on average $0.35 \pm$ $0.02 \mathrm{mV}$. The antennae of both species gave the highest responses to nonanal $(87 \%$ of the standard at $0.1 \mathrm{M})$.
Caryophyllene and $\alpha$-pinene gave only weak responses ( $22 \%$ of the standard), but still significantly higher responses than the control. In Fig. 1 an overview of the responses to all stimuli (at $0.01 \mathrm{M}$ ) (normalised to the standard) is presented for both species. Two acetates (decyl acetate and dodecyl acetate) evoked significantly higher responses in B. terrestris. An analysis of variance showed significant main effects of concentration and species, as well as significant stimulus*species interactions (Table 1). Inspection of the individual coefficients for this interactions showed that the two acetates were the only two compounds that produced significantly different responses in workers of A. mellifera and workers of $B$.terrestris, while the other compounds showed a similar reaction in both species (Table 2). In Fig. 2 the dose-response curves for nonanal and the two acetates are presented, again demonstrating the clear species difference for the two acetates.

\section{Discussion}

We found a significant difference in antennal reaction towards two acetates, decyl acetate and dodecyl acetate, 
between workers of Apis mellifera and workers of Bombus terrestris. Dodecyl acetate caused the greatest disparity in reaction. A significantly stronger EAG response in B.terrestris workers likely indicates a more important role for the two acetates in attraction of bumblebees than in honeybees. The floral scent of $C$. calceolus contains large quantities of acetates (Claessens and Kleynen, 2011), in particular octyl acetate, decyl acetate and dodecyl acetate (Bergström et al., 1992). In addition, pheromones employed by some species of Andrenidae and bumblebees include dodecyl acetate (Tengö and Bergström, 1977; Coppée et al., 2008). Males of solitary bees odour-mark nest entrances or flowers using acetates in order to attract females, attracting other males at the same time (Schmitt and Bertsch, 1990). Dodecyl acetate has not been reported as a part of honeybee's pheromones. Therefore, our finding shows that EAG responses can be a first indication of a difference in importance of different chemicals in the biology of bees.

Our EAG findings that dodecyl acetate likely plays a more important role in the biology of bumblebees than in the biology of honeybees seems to have some confirmation in field observations. Nilsson (1979) reported seven different species of bumblebees $(15 \%$ of all visitors), but not honeybees, visiting (although not pollinating) European slipper orchids. The bumblebees in the geographical area of the study were too large to be effective pollinators and could easily climb out the main entrance of the flowers (Nilsson, 1979). Nevertheless, they seem to be attracted to C.calceolus. This suggests that dodecyl acetate plays a role in attracting some bee species (bumblebees), but not others (honeybees).

Cephalic labial gland secretions play an important role as sex pheromones of some bumblebees species, including subspecies of $B$. terrestris. Those secretions include dodecyl acetate (Bergman and Bergström, 1997; Hovorka et al., 2006; Coppée et al, 2008, 2011). Since in the current study dodecyl acetate induces the greatest disparity in EAG response between $B$. terrestris and $A$. mellifera (Fig. 1), this suggests that $C$. calceolus might use mimicry specifically targeting bumblebee chemical communication.

The EAG technique used only reveals whether an insect is capable of detecting chemical compounds; the response (i.e. attraction or repulsion) cannot be inferred without additional behavioural studies. Additional experiments with the natural pollinators are needed to reveal whether $C$. calceolus indeed exploits floral fragrance similar to the orchid genera Ophrys L. (sexual deception; Schiestl et al., 2010) and Epipactis Zinn (brood-site mimicry; Stökl et al., 2010). Natural pollinators of $C$. calceolus are sand bees belonging to the Andrenidae and Halictidae (Daumann, 1968; Nilsson, 1979). Müller (1873) recorded female Andrena albicans (Müller, 1796), A. flavipes (Panzer, 1799), A. fulvicrus (Kirby, 1802), A. nigroaenea (Kirby, 1802), A. ovina (Klug, 1810) and A. tibialis (Kirby, 1802) transferring pollinia in Germany. On the island of Öland (Sweden), the medium-sized A. haemorrhoa (Fabricius, 1781) was the most important pollinator (Nilsson 1979). On the mainland of Sweden, Antonelli et al. (2009) recorded A. carantonica (Pérez, 1902), A. cineraria (Linneaus, 1508), A. helvola (Linneaus, 1758), and A. praecox (Scopoli, 1763) as pollinators in addition to Colletes cunicularis (Linneaus, 1761), Lasioglossum fratellum (Perez, 1903) and L. fulvicorne (Kirby, 1802). In Denmark Halictus tumulorum (Linnaeus, 1508) and Lasioglossum calceatum (Scopoli, 1763) were the most regular pollinators (Erneberg and Holm, 1999). On the British Isles, Andrena scotica (Perkins, 1916) was recorded as a pollinator (Kull, 1999). Experiments carried out with the natural pollinators of $C$. calceolus so far revealed that farnesol and farnesyl hexanoate inhibit copulation behaviour of males of A. nigroaenea (Schiestl and Ayasse, 2010). Furthermore, $n$-alkanes and $n$-alkenes triggered copulation behaviour of males of A. nigroaenea (Schiestl et al., 2000). These compounds are not present in the floral fragrance of $C$. calceolus, so if mimicry is employed by this orchid species, other pheromones are targeted.

\section{Conclusions and further study}

The findings of our current EAG experiment showed that antennae of B. terrestris workers respond to decyl acetate and dodecyl acetate significantly more than the antennae of A. mellifera, with response to dodecyl acetate being the highest. This suggests that dodecyl acetate plays a more important role in the biology of bumblebees than in the biology of honeybees. Our study is the first study that involved an EAG experiment using volatiles found in C. calceolus flowers. Although bumblebees and honeybees are good models for studying some aspects of bee sensory responses, the mechanisms used by this orchid species to lure visitors should now be investigated further using sand bees that are the natural pollinators of $C$. calceolus. After understanding the effects of the chemical signals 
employed by this orchid species, we will hopefully be able to disentangle the role of visual cues provided by its floral asymmetry as well.

\section{Acknowledgements}

We would like to thank Vivek Nityananda for the initial introduction to bumblebee behavioural experimental techniques, Lina Kawaguchi for her feedback on bumblebee colony management, Ludek Tikovsky for his help with the logistics in the greenhouse complex of the University of Amsterdam, Rene Genet for allowing us to collect honeybees from his beehive in Diemerpark and two anonymous reviewers for helping us improving earlier versions of this manuscript.

\section{References}

Ackerman JD. 1986. Mechanisms and evolution of food-deceptive pollination systems in orchids. Lindleyana 1: 108-113.

Antonelli A, Dahlberg CJ, Carlgren KHI, Appelqvist T. 2009. Pollination of the Lady's slipper orchid (Cypripedium calceolus) in Scandinavia taxonomic and conservational aspects. Nordic Journal of Botany 27: 266-273.

Appelgren M, Bergström G, Svensson BG, Cederberg B. 1991. Marking pheromones of Megabombus bumble bee males. Acta Chemica Scandinavica 45: 972-974.

Baer B. 2003. Bumblebees as model organisms to study male sexual selection in social insects. Behavioral Ecology and Sociobiology 54: 521-533.

Barkman, TJ, Beaman H, Gaget DA. 1997. Floral fragrance variation in Cypripedium: Implication for evolutionary and ecological studies. Phytochemistry 44: 875-882.

Bell AK, Roeberts DL, Hawkings JA, Rudall PJ, Box MS, Bateman RM. 2009. Comparative micromorphology of nectariferous and nectarless labellar spurs in selected clades of subtribe Orchidinae (Orchidaceae). Botanical Journal of the Linnean Society 160: 369-387.

Benard J, Stach S, Giurfa M. 2006. Categorization of visual stimuli in the honeybee Apis mellifera. Animal Cognition 9: 257-270.

Bergman P, Bergström G. 1997. Scent marking, scent origin, and species specificity in male premating behavior of two Scandinavian bumblebees. Journal of Chemical Ecology 5: 1235-1251.

Bergström G, Birgersson G, Groth I, Nilsson LA. 1992. Floral fragrance disparity between three taxa of Lady's Slipper Cypripedium calceolus (Orchidaceae). Phytochemistry 31: 2315-2319.

Bertsch A, Schweer H, Titze A. 2004. Analysis of the labial gland secretions of the male bumblebee Bombus griseocollis (Hymenoptera: Apidae). Zeitschrift für Naturforschung 59c: 701-707.

Cane JH, Tengo J. 1981. Pheromonal cues direct mate-seeking behavior of male Colletes cunicularius (Hymenoptera: Colletidae). Journal of Chemical Ecology 7: 427-436.

Case MA, Bradford ZR. 2009. Enhancing the trap of lady's slippers: a new technique for discovering pollinators yields new data from Cypripedium parviflorum (Orchidaceae). Botanical Journal of the Linnean Society 160: 1-10.

Chittka L. 1996. Does bee colour vision predate the evolution of flower colour? Naturwissenschaften 83: 136-138.

Chittka L, Beier W, Hertel H, Steinmann E, Menzel R. 1992. Opponent colour coding is a universal strategy to evaluate the photoreceptor inputs in hymenoptera. Journal of Comparative Physiology A 170: 545-563.

Claessens J, Kleynen J. 2011. The flower of the European orchid. Form and function. Voerendaal/Stein: Schrijen-Lippertz.

Coppée A, Terzo M, Valterova I, Rasmont P. 2008. intraspecific variation of the cephalic labial gland secretions in Bombus terrestris (L.) (Hymenoptera: Apidae). Chemistry and Biodiversity 5: 2654-2661.

Coppée A, Mathy T, Cammaerts MC,Vergeggen FJ, Terzo M Iserbyt S, Valterová I, Rasmont P. 2011. Age-dependent attractivity of males' sexual pheromones in Bombus terrestris (L.) [Hymenoptera, Apidae]. Chemoecology 21: 75-82.

Cozzolino S, Widmer A. 2005. Orchid diversity: an evolutionary consequence of deception? Trends in Ecology and Evolution 20: 487-494.

Dafni A. 1984. Mimicry and deception in pollination. Annual Review of Ecology, Evolution and Systematics 15: 259278.

Darwin C. 1877. The various contrivances by which orchids are fertilised by insects. 2nd ed. London: J. Murray.

Daumann E. 1968. Zur Bestaubungsbkologie von Cypripedium calceolus L. Österreichische Botanische Zeitschrift 115: 434-446.

Dyer AG, Chittka L. 2004. Fine colour discrimination requires differential conditioning in bumblebees. Naturwissenschaften 91: 224-227.

Erneberg M, Holm B. 1999. Bee size and pollen transfer in Cypripedium calceolus (Orchidaceae). Nordic Journal of Botany 19: 363-367.

Ferdy JB, Gouyon PH, Moret J, Godelle B. 1998. Pollinator behavior and deceptive pollination: learning process and floral evolution. The American Naturalist 152: 696-705.

Giurfa M, Dafni A, Neal PR. 1999. Floral symmetry and its role in plant-pollinator systems. International Journal of Plant Sciences 160: S41-S50.

Granero AM, Guerra Sanz JM, Egea Gonzalez FJ, Martinez Vidal JL, Dornhaus A, Ghani J, Roldán Serrano A, Chittka, L. 2005. Chemical compounds of the foraging recruitment pheromone in bumblebees. Naturwissenschaften 92: 371374.

Hornik, K. 2011. The R FAQ. http://CRAN.R-project.org/doc/ FAQ/R-FAQ.html

Hovorka O, Valterová I, Rasmont P, Terzo M. 2006. Male cephalic labial gland secretions of two bumblebee species of the subgenus Cullumanobombus (Hymenoptera: Apidae: Bombus Latreille) and their distribution in Central Europe. Chemistry and Biodiversity 3: 1015-1022.

Jersáková J, Johnson SD, Kindlmann P. 2006. Mechanisms and evolution of deceptive pollination in orchids. Biological Reviews 81: 219-235.

Lotto RB, Chittka L. 2005. Seeing the light: Illumination as a contextual cue to color choice behavior in bumblebees. Proceedings of the National Academy of Sciences 102: 3852-3856. 
Kull T. 1999. Biological flora of the British Isles. Cypripedium calceolus L. Journal of Ecology 87: 913-924.

Müller H. 1873. The fertilisation of flowers. London: Macmillan.

Nilsson LA. 1979. Anthecological studies on the Lady's Slipper, Cypripedium calceolus (Orchidaceae). Botaniska notiser 132: 329-347.

Raine NE, Chittka L. 2008. The correlation of learning speed and natural foraging success in bumble-bees. Proceedings of the Royal Society - Series B 275: 803-808.

Rodríguez I, Gumbert A, Hempel de Ibarra N, Kunze J, Giurfa M. 2004. Symmetry is in the eye of the 'beeholder': innate preference for bilateral symmetry in flower-naïve bumblebees. Naturwissenschaften 91: 374-377.

Schiestl FP. 2005. On the success of a swindle: pollination by deception in orchids. Naturwissenschaften 92: 255-264.

Schiestl FP. 2010. Pollination: sexual mimicry abounds. Current Biology 20: R1020-R1022.

Schiestl FP, Ayasse M, Pauls HF, Lofstedt C, Hanson BS, Ibarra F, Francke W. 2000. Sex pheromone mimicry in the early spider orchid (Ophrys sphegodes): patterns of hycrocarbons and the key mechanism for pollination by sexual selection. Journal of Comparative Physiology A. Neuroethology, Sensory, Neural and Behavioral Physiology 186: 567574.

Schmitt U, Bertsch A. 1990. Do foraging bumblebees scentmark food sources and does it matter? Oecologia 82: 137144.

Scopece G, Cozzolino S, Johnson SD, Schiestl FP. 2010. Pollination efficiency and the evolution of specialized deceptive pollination systems. The American Naturalist 175: 98-105.
Schneider D. 1957. Elektrophysiologische Untersuchungen von Chemo- und Mechanorezeptoren der Antenne des Seidenspinners (Bombyx mori L.). Zeitschrift für Vergleichende Physiologie 40: 8-41.

Sprengel CK. 1793. Das Entdeckte Geheimniss in der Natur im Bau und in der Befuchtung der Blumen. (Reprinted 1972). New York: Weldon and Wesley.

Stökl J, Brodmann J, Dafni A, Ayasse M, Hansson BS. 2010. Smells like aphids: orchid flowers mimic aphid alarm pheromones to attract hoverflies for pollination. Proceedings of the Royal Society B 278: 1216-1222.

Tengö J, Bergström G. 1977. Comparative analyses of complex secretions from heads of Andrena bees (Hym., Apoidea). Comparative Biochemistry 57: 197-202.

Urbaniak GC, Plous S. 2011. Research Randomizer v. 3.0. Available from http: //www.randomizer.org/

Waser NM, Chittka L, Price MV, Williams N, Ollerton J. 1996. Generalization in pollination systems, and why it matters. Ecology 77: 1043-1060.

Welch CJ. 1998. Some observations concerning the asymmetry of orchid flowers. Malayan Orchid Review 32: 86-92.

Welch CJ. 2002. Chirality in the natural world: life through the looking glass. Pp. 285-302 in: Lough WJ, Wainer IW, eds, Chirality in the natural and applied sciences. Oxford: Blackwell.

Received: 16 December 2011

Revised and accepted: 2 April 2012

Published online: 31 May 2012

Editor: M. Schilthuizen 